\title{
Optimal Control for Proportional Economic Growth
}

\author{
A. V. Kryazhimskii ${ }^{\dagger}$ and A. M. Taras'ev ${ }^{1}$
}

Received February 16, 2015

\begin{abstract}
The research is focused on the question of proportional development in economic growth modeling. A multilevel dynamic optimization model is developed for the construction of balanced proportions for production factors and investments in a situation of changing prices. At the first level, models with production functions of different types are examined within the classical static optimization approach. It is shown that all these models possess the property of proportionality: in the solution of product maximization and cost minimization problems, production factor levels are directly proportional to each other with coefficients of proportionality depending on prices and elasticities of production functions. At the second level, proportional solutions of the first level are transferred to an economic growth model to solve the problem of dynamic optimization for the investments in production factors. Due to proportionality conditions and the homogeneity condition of degree 1 for the macroeconomic production functions, the original nonlinear dynamics is converted to a linear system of differential equations that describe the dynamics of production factors. In the conversion, all peculiarities of the nonlinear model are hidden in a time-dependent scale factor (total factor productivity) of the linear model, which is determined by proportions between prices and elasticities of the production functions. For a control problem with linear dynamics, analytic formulas are obtained for optimal development trajectories within the Pontryagin maximum principle for statements with finite and infinite horizons. It is shown that solutions of these two problems differ crucially from each other: in finite horizon problems the optimal investment strategy inevitably has the zero regime at the final stage, whereas the infinite horizon problem always has a strictly positive solution. A remarkable result of the proposed model consists in constructive analytical solutions for optimal investments in production factors, which depend on the price dynamics and other economic parameters such as elasticities of production functions, total factor productivity, and depreciation factors. This feature serves as a background for the productive fusion of optimization models for investments in production factors in the framework of a multilevel structure and provides a solid basis for constructing optimal trajectories of economic development.
\end{abstract}

Keywords: optimal control, Pontryagin maximum principle, multilevel optimization, proportional economic growth.

DOI: $10.1134 / \mathrm{S} 0081543816050102$

\section{INTRODUCTION}

The paper is devoted to the construction and analysis of the model of proportional development within the theory of economic growth. The proposed approach combines elements of

\footnotetext{
${ }^{\dagger}$ Deceased.

${ }^{1}$ Krasovskii Institute of Mathematics and Mechanics, Ural Branch of the Russian Academy of Sciences, ul. S. Kovalevskoi 16, Yekaterinburg, 620990 Russia; International Institute for Applied Systems Analysis, Schlossplatz 1, A-2361 Laxenburg, Austria

e-mail: tam@imm.uran.ru, tarasiev@iiasa.ac.at
} 
the classical models [1-5] and the ideas of the proportionality of optimal solutions of static microeconomic and macroeconomic models [6] with the dynamic optimization constructions in the Pontryagin maximum principle [7] and its generalizations for optimal control problems with infinite horizon [8-11].

The model implements the results of investigating the optimization of investments in production factors [12-21] and essentially supplements and renews them by applying multilevel optimization constructions.

It should be noted that similar approaches to studying economic growth models based on proportional constructions were developed in [22].

The main idea of the paper consists in designing an economic growth model with multilevel optimization constructions.

At the first level, we consider an optimization procedure for a static problem if the current time period is fixed. Here, two standard statements are possible: the cost minimization problem with a fixed production level and the dual product maximization problem with specified costs. For models with the classical exponential Cobb-Douglas production functions, production functions with constant elasticity of substitution, and input-output production functions [6], it is shown that solutions of both static optimization problems possess the properties of proportionality: proportions between optimal production factors are determined by proportions between prices and elasticities of the production functions.

At the second level, we study an optimal control problem under proportionality conditions within the theory of economic growth. To pass to this problem, the solutions of the first optimization level are substituted into the equations of production factor dynamics. In such a passage, the homogeneity property of degree 1 for the production function (the property of constant return to scale) combined with the proportionality properties of the production factors generate a linear dynamics of production factors and costs.

An optimal control problem is posed for an integral utility function with logarithmic consumption index on trajectories of the obtained linear system describing the dynamics of production factors. Note that the use of the logarithmic consumption index is a basic construction in the theory of endogenous economic growth $[3,6]$. In addition, a utility function of this type is closely related to the notion of entropy of a dynamic system.

The solution of the optimal control problem is obtained within the Pontryagin maximum principle [7] for finite horizon and its generalizations for statements with infinite horizon [8]. An important property of the solution is that it is given by analytic formulas for a wide range of model parameters including prices and elasticities depending on time. Note that such a time dependence can have a complicated character and can provide for growth trends, transition periods, cycles, and crises in the model.

It is worth noting that analytic solutions for the optimal control are obtained in both problems, with finite and infinite horizons. The structure of these solutions clearly shows that the optimal control always has a zero investments regime at the final stage in the problem with finite horizon. In contrast to this case, the solution of the problem with infinite horizon may have no zero values for the optimal investments on the whole time interval.

For the first and second optimization levels, a feedback is established by transferring the optimal solution from the second level to the first one, where the investments are redistributed between production factors in accordance with the proportionality principle.

Based on the proposed approach, optimal analytic solutions are constructed for both opti-

$$
\text { PROCEEDINGS OF THE STEKLOV INSTITUTE OF MATHEMATICS } \quad \text { Vol. } 293 \quad \text { Suppl. } 1 \quad 2016
$$


mization levels. The corresponding optimal levels of investment generate a system of differential equations for the production factors similar to the replicator dynamics of evolutionary games [23,24]. It should be noted that, in the context mentioned above, the optimal solutions obtained can be analyzed within the theory of positional differential games [25].

Due to the effective construction, the proposed technique of multilevel optimization can be applied to the econometric identification and prognostic simulation of optimal scenarios of proportional growth in multidimensional economic systems.

The paper is organized as follows.

In the beginning, we form the first level of the model optimization, at which the time period is fixed.

In Section 1, we consider the cost minimization problem with a fixed production level for models with the classical production functions: exponential Cobb-Douglas functions and functions with constant elasticity of substitution. It is proved that the solutions of these problems possess the proportionality property: the optimal costs of production factors should be proportional to each other with coefficients depending on proportions between prices and elasticities.

In Section 2, we analyze the product maximization problem, which is dual to the cost minimization problem. Similar proportions of optimal solutions are obtained for the statements with the Cobb-Douglas production functions and functions with constant elasticity of substitution.

In Section 3, proportionality conditions are naturally derived also for the models with inputoutput production functions.

In Section 4, we establish connections between the optimal production and costs. Under the condition of constant return to scale (the unit production elasticity), it is shown that the optimal production can be linearly expressed in terms of costs with a scale coefficient specified by proportions between prices and elasticities. Therefore, nonlinear dependencies in the production functions can be hidden in the structure of the scale coefficient and can be expressed in terms of current proportions.

In Section 5, we introduce balance equations for consumption and investment in both absolute and relative variables. This construction completes the first optimization level for the model of proportional economic growth.

Then, we pass to the second optimization level, where the time period is unlocked and becomes the main variable.

In Section 6, the dynamics of production factors is given by a system of differential equations describing the influence of investments as control parameters on the economic development. We also introduce a utility function for the economic growth in the integral form (on the finite or infinite horizon) of the discounted logarithmic consumption index defined on trajectories of the system. It is proved that there exists a possibility to redistribute investments in the current time period in such a way that the proportions of optimal solutions of the cost minimization or product maximization problems at the first model level are sustained. It should be noted that the obtained system of differential equations for the dynamics of production factors has characteristic features of the replicator dynamics from the theory of evolution games.

In Section 7, at the second model optimization level, the optimal control problem for the integral logarithmic consumption index is posed on trajectories of the linear cost dynamics. For this problem, the Pontryagin maximum principle is formulated for both statements (with finite and infinite horizons).

In Section 8, we analyze the Hamiltonian systems arising in the Pontryagin maximum principle PROCEEDINGS OF THE STEKLOV INSTITUTE OF MATHEMATICS $\quad$ Vol. $293 \quad$ Suppl. $1 \quad 2016$ 
and construct optimal solutions. Analytical formulas for the optimal control are derived for the problems with finite and infinite horizons. Sufficient optimality conditions based on the concavity properties of the maximized Hamiltonian are specified for the obtained solutions. It is shown that the optimal control structure in the problem with finite horizon inevitably has the zero regime at the final stage. On the contrary, the optimal control in the problem with infinite horizon is, as a rule, strictly positive on the whole time interval. This result shows the advantages of the optimal control problems with infinite horizon in models of economic growth in comparison with the problems with finite horizon.

\section{PROPORTIONALITY IN THE COST MINIMIZATION PROBLEM}

In this section, we study the cost minimization problems of the first level for models with production functions of different types. It is shown that the solutions of these problems possess the property of proportionality: the production factor levels are directly proportional to each other with coefficients of proportionality depending on prices and elasticities of the production functions.

At the first level, we fix a time period $t, t \in\left[t_{0}, T\right], t_{0} \leq T \leq+\infty$, and consider static optimization problems. To simplify the computations, we omit the time symbol $t$. However, one should keep in mind that all the parameters and model variables may depend on time.

1.1. Cost minimization for the Cobb-Douglas production function. Let us consider an economy model equipped with a Cobb-Douglas production function

$$
y=a \prod_{j=1}^{n} x_{j}^{\alpha_{j}} .
$$

Here, $y$ is the production level, and the symbols $x_{1}, \ldots, x_{n}$ denote the sizes of the production factors $1, \ldots, n$, respectively. The parameter $a, a>0$, plays the role of a scale coefficient (the total factor productivity). For the elasticities $\alpha_{j}$ of the production function with respect to the factors, we assume that the following conditions are fulfilled:

$$
\alpha_{1}, \ldots, \alpha_{n}>0, \quad \sum_{j=1}^{m} \alpha_{j}=\varepsilon
$$

Here, the parameter $\varepsilon$ stands for the elasticity of production with respect to the investment. In macroeconomic models, as a rule, it is assumed that the constant return to scale is used in the form of the homogeneity of degree 1 for the production function; i.e., the elasticity of production is equal to one, $\varepsilon=1$.

Let us introduce the prices of the production factors. The symbol $p_{j}, p_{j}>0$, stands for the price of the unit of production factor $j$.

Consider the cost minimization problem with a fixed production level. It is required to find optimal values of the production factors $x_{1}, \ldots, x_{n}$ that minimize the total costs $C$ :

$$
\text { Minimize } \quad C=\sum_{j=1}^{n} p_{j} x_{j}
$$

under the following constraints on a fixed production level $y>0$ :

$$
x_{1}, \ldots, x_{n} \geq 0, \quad y=a \prod_{j=1}^{n} x_{j}^{\alpha_{j}}
$$


Here, it is convenient to pass to the logarithmic type constraints $\ln y=\ln a+\alpha_{1} \ln x_{1}+\ldots+a_{n} \ln x_{n}$.

According to the method of Lagrange multipliers, one can construct the Lagrange function

$$
L\left(x_{1}, \ldots, x_{n}, \lambda\right)=p_{1} x_{1}+\ldots+p_{n} x_{n}+\lambda\left(\ln y-\ln a-\alpha_{1} \ln x_{1}-\ldots-a_{n} \ln x_{n}\right) .
$$

Here, the parameter $\lambda$ denotes the Lagrange multiplier corresponding to the logarithmic constraints for the Cobb-Douglas production function.

The necessary optimality conditions in the cost minimization problem imply the following system of relations:

$$
\frac{\partial L}{\partial x_{j}}=p_{j}-\lambda \frac{\alpha_{j}}{x_{j}}=0, \quad j=1, \ldots, n,
$$

which provide the current proportions between the production factors:

$$
c_{i k}=\frac{x_{i}}{x_{k}}=\frac{\alpha_{i} p_{k}}{\alpha_{k} p_{i}}, \quad i=1, \ldots, n, \quad k=1, \ldots, n .
$$

It is possible to derive the values of demand functions for the production factors in the following form:

$$
x_{j}=\left(\frac{y}{a}\right)^{\frac{1}{\varepsilon}}\left(\frac{\alpha_{j}}{p_{j}}\right) \prod_{k=1}^{n}\left(\frac{p_{k}}{\alpha_{k}}\right)^{\frac{\alpha_{k}}{\varepsilon}}, \quad j=1, \ldots, n .
$$

In the case of the constant return to scale, $\varepsilon=1$, we obtain the following expressions for the demand functions:

$$
x_{j}=\frac{y}{a}\left(\frac{\alpha_{j}}{p_{j}}\right) \prod_{k=1}^{n}\left(\frac{p_{k}}{\alpha_{k}}\right)^{\alpha_{k}}, \quad j=1, \ldots, n .
$$

1.2. Cost minimization for the CES production function. Consider a model based on a production function with constant elasticity of substitution (CES):

$$
y=e_{0}\left(e_{1} x_{1}^{-\beta}+\ldots+e_{n} x_{n}^{-\beta}\right)^{-\frac{h}{\beta}} .
$$

Here, the parameter $e_{0}>0$ specifies the total factor productivity, the parameters $e_{j} \geq 0, j=$ $1, \ldots, n$, are share coefficients, the parameter $h>0$ defines the degree of homogeneity of the production function, and the parameter $\beta, \beta>-1$, is the coefficient of substitution. The elasticity of production is defined by the degree of homogeneity $h, \varepsilon=h$. For the constant return to scale, the condition $h=1$ should be fulfilled.

Let us analyze the cost minimization problem for the CES production function. In this case, the Lagrange function is of the form

$$
L\left(x_{1}, \ldots, x_{n}, \lambda\right)=p_{1} x_{1}+\ldots+p_{n} x_{n}+\lambda\left(\ln y-\ln e_{0}+\frac{h}{\beta} \ln \left(e_{1} x_{1}^{-\beta}+\ldots+e_{n} x_{n}^{-\beta}\right)\right) .
$$

The necessary optimality conditions in the cost minimization problem are given by the relations

$$
\frac{\partial L}{\partial x_{j}}=p_{j}-\lambda \frac{h}{\beta} \frac{-\beta e_{j} x_{j}^{-(\beta+1)}}{\left(e_{1} x_{1}^{-\beta}+\ldots+e_{n} x_{n}^{-\beta}\right)}=0, \quad j=1, \ldots, n .
$$

These conditions provide the proportionality for the production factors

$$
c_{i k}=\frac{x_{i}}{x_{k}}=\left(\frac{e_{i} p_{k}}{e_{k} p_{i}}\right)^{\frac{1}{(\beta+1)}}, \quad i=1, \ldots, n, \quad k=1, \ldots, n .
$$


Using these conditions, we derive the demand functions for the production factors in the model with the CES production function:

$$
x_{j}=\left(\frac{y}{e_{0}}\right)^{\frac{1}{h}}\left(\frac{e_{j}}{p_{j}}\right)^{\frac{1}{(\beta+1)}}\left(e_{1}^{\frac{1}{(\beta+1)}} p_{1}^{\frac{\beta}{(\beta+1)}}+\ldots+e_{n}^{\frac{1}{(\beta+1)}} p_{n}^{\frac{\beta}{(\beta+1)}}\right)^{\frac{1}{\beta}}, \quad j=1, \ldots, n .
$$

For the constant return to scale, $h=1$, the following relations for the demand functions are obtained:

$$
x_{j}=\frac{y}{e_{0}}\left(\frac{e_{j}}{p_{j}}\right)^{\frac{1}{(\beta+1)}}\left(e_{1}^{\frac{1}{(\beta+1)}} p_{1}^{\frac{\beta}{(\beta+1)}}+\ldots+e_{n}^{\frac{1}{(\beta+1)}} p_{n}^{\frac{\beta}{(\beta+1)}}\right)^{\frac{1}{\beta}}, \quad j=1, \ldots, n .
$$

1.3. Sufficient optimality conditions for proportional solutions. In order to establish sufficient optimality conditions for the proportional solutions obtained, one should calculate the matrix of second derivatives (the Hessian matrix) of the Lagrange function $L(x, \lambda)$ with respect to the production factors $x$ and then verify that this matrix is positive definite.

For the model with Cobb-Douglas production function (1.1), we have the matrix of second derivatives

$$
\frac{\partial^{2} L}{\partial x_{j}^{2}}=\frac{\lambda \alpha_{j}}{x_{j}^{2}}>0, \quad \frac{\partial^{2} L}{\partial x_{j} \partial x_{k}}=0, \quad j=1, \ldots, n, \quad j=1, \ldots, n, \quad k \neq j .
$$

Obviously, this diagonal matrix satisfies the Sylvester criterion and, consequently, is positive definite.

In the model with CES production function (1.3), the matrix of second derivatives has the following structure:

$$
\begin{gathered}
\frac{\partial^{2} L}{\partial x_{j}^{2}}=\frac{\lambda h e_{j}\left((\beta+1) x_{j}^{-(\beta+2)}\left(\sum_{k \neq j} e_{k} x_{k}^{-\beta}\right)+e_{j} x_{j}^{-2(\beta+1)}\right)}{\left(e_{1} x_{1}^{-\beta}+\ldots+e_{n} x_{n}^{-\beta}\right)^{2}}>0, \\
\frac{\partial^{2} L}{\partial x_{j} \partial x_{k}}=-\frac{\lambda h \beta e_{j} x_{j}^{-(\beta+1)} e_{k} x_{k}^{-(\beta+1)}}{\left(e_{1} x_{1}^{-\beta}+\ldots+e_{n} x_{n}^{-\beta}\right)^{2}}<0, \\
j=1, \ldots, n, \quad j=1, \ldots, n, \quad k \neq j .
\end{gathered}
$$

For this matrix, it is also possible to verify that the Sylvester criterion holds and the matrix is positive definite. For example, in the case of two production factors for $n=2$, the determinant of the matrix of second derivatives of the Lagrange function is positive:

$$
\Delta=\frac{\partial^{2} L}{\partial x_{1}^{2}} \frac{\partial^{2} L}{\partial x_{2}^{2}}-\left(\frac{\partial^{2} L}{\partial x_{1} \partial x_{2}}\right)^{2}=\frac{\lambda^{2} h^{2}(\beta+1) e_{1} e_{2} x_{1}^{-(\beta+2)} x_{2}^{-(\beta+2)}}{\left(e_{1} x_{1}^{-\beta}+e_{2} x_{2}^{-\beta}\right)^{2}}>0,
$$

and, consequently, the matrix of second derivatives is positive definite.

Thus, for both classical production functions, sufficient minimum conditions for proportional solutions are fulfilled.

\section{PROPORTIONALITY IN THE PRODUCT MAXIMIZATION PROBLEM}

Proportionality conditions take place not only for the cost minimization problem but also for the dual product maximization problem. 
2.1. Maximal solutions for the Cobb-Douglas production function. In the model with a Cobb-Douglas production function, it is required to maximize the product

$$
y=a x_{1}^{\alpha_{1}} \ldots x_{n}^{\alpha_{n}} \longrightarrow \max
$$

under cost constraints

$$
p_{1} x_{1}+\ldots+p_{n} x_{n}=C
$$

Here, the parameter $C, C>0$, denotes the total costs.

The Lagrange function in the product maximization problem has the form

$$
L\left(x_{1}, \ldots, x_{n}, \lambda\right)=a x_{1}^{\alpha_{1}} \ldots x_{n}^{\alpha_{n}}+\lambda\left(C-p_{1} x_{1}-\ldots-p_{n} x_{n}\right) .
$$

In this case, the necessary optimality conditions generate the system of equations

$$
\frac{\partial L}{\partial x_{j}}=a \alpha_{j} x_{j}^{-1} x_{1}^{\alpha_{1}} \ldots x_{n}^{\alpha_{n}}-\lambda p_{j}=0, \quad j=1, \ldots, n .
$$

The Lagrange multiplier is represented by the relation $\lambda=\frac{\alpha_{1}}{p_{1} x_{1}} y=\ldots=\frac{\alpha_{n}}{p_{n} x_{n}} y$.

The latter conditions, in turn, imply the proportionality properties in the production maximization problem:

$$
c_{i k}=\frac{x_{i}}{x_{k}}=\frac{\alpha_{i} p_{k}}{\alpha_{k} p_{i}}, \quad i=1, \ldots, n, \quad k=1, \ldots, n .
$$

These properties are similar to the proportionality properties in cost minimization problem (1.2).

In this case, we obtain the following optimal solutions for the demand functions:

$$
x_{j}=C \frac{\alpha_{j}}{p_{j}}, \quad j=1, \ldots, n .
$$

In addition, the values of optimal production are derived from the given costs:

$$
y=a C\left(\frac{\alpha_{1}}{p_{1}}\right)^{\alpha_{1}} \ldots\left(\frac{\alpha_{n}}{p_{n}}\right)^{\alpha_{n}}=a x_{j} \frac{p_{j}}{\alpha_{j}}\left(\frac{\alpha_{1}}{p_{1}}\right)^{\alpha_{1}} \ldots\left(\frac{\alpha_{n}}{p_{n}}\right)^{\alpha_{n}}, \quad j=1, \ldots, n .
$$

2.2. Maximal solutions for the CES production function. Consider the product maximization problem for a CES production function:

$$
y=e_{0}\left(e_{1} x_{1}^{-\beta}+\ldots+e_{n} x_{n}^{-\beta}\right)^{-\frac{h}{\beta}} \longrightarrow \max
$$

under cost constraints (2.1).

In this case, the Lagrange function has the form

$$
L\left(x_{1}, \ldots, x_{n}, \lambda\right)=e_{0}\left(e_{1} x_{1}^{-\beta}+\ldots+e_{n} x_{n}^{-\beta}\right)^{-\frac{h}{\beta}}+\lambda\left(C-p_{1} x_{1}-\ldots-p_{n} x_{n}\right) .
$$

The necessary optimality conditions in the product maximization problem with the CES production function are given by the relations

$$
\frac{\partial L}{\partial x_{j}}=e_{0}\left(-\frac{h}{\beta}\right)\left(e_{1} x_{1}^{-\beta}+\ldots+e_{n} x_{n}^{-\beta}\right)^{-\frac{h}{\beta}-1} e_{j}(-\beta) x_{j}^{-(\beta+1)}-\lambda p_{j}=0, \quad j=1, \ldots, n .
$$


These relations imply the proportionality conditions for the CES production function in the product maximization problem:

$$
c_{i k}=\frac{x_{i}}{x_{k}}=\left(\frac{e_{i} p_{k}}{e_{k} p_{i}}\right)^{\frac{1}{(\beta+1)}}, \quad i=1, \ldots, n, \quad k=1, \ldots, n
$$

These conditions are completely identical to the proportionality conditions in the cost minimization problem, see (1.4).

The demand functions for the production factors are obtained in the form of the relations

$$
x_{j}=C\left(\frac{e_{j}}{p_{j}}\right)^{\frac{1}{(\beta+1)}}\left(e_{1}^{\frac{1}{(\beta+1)}} p_{1}^{\frac{\beta}{(\beta+1)}}+\ldots+e_{n}^{\frac{1}{(\beta+1)}} p_{n}^{\frac{\beta}{(\beta+1)}}\right)^{-1}, \quad j=1, \ldots, n
$$

To represent the optimal production in terms of the given costs, we derive the formula

$$
\begin{aligned}
y & =e_{0} C^{h}\left(e_{1}^{\frac{1}{(\beta+1)}} p_{1}^{\frac{\beta}{(\beta+1)}}+\ldots+e_{n}^{\frac{1}{(\beta+1)}} p_{n}^{\frac{\beta}{(\beta+1)}}\right)^{-h \frac{(\beta+1)}{\beta}} \\
& =e_{0} x_{j}^{h}\left(\frac{p_{j}}{e_{j}}\right)^{\frac{h}{(\beta+1)}}\left(e_{1}^{\frac{1}{(\beta+1)}} p_{1}^{\frac{\beta}{(\beta+1)}}+\ldots+e_{n}^{\frac{1}{(\beta+1)}} p_{n}^{\frac{\beta}{(\beta+1)}}\right)^{\frac{h}{\beta}}, \quad j=1, \ldots, n .
\end{aligned}
$$

In the case of the constant return to scale, $h=1$, we have the following relations for the optimal production:

$$
\begin{aligned}
y & =e_{0} C\left(e_{1}^{\frac{1}{(\beta+1)}} p_{1}^{\frac{\beta}{(\beta+1)}}+\ldots+e_{n}^{\frac{1}{(\beta+1)}} p_{n}^{\frac{\beta}{(\beta+1)}}\right)^{-\frac{(\beta+1)}{\beta}} \\
& =e_{0} x_{j}\left(\frac{p_{j}}{e_{j}}\right)^{\frac{1}{(\beta+1)}}\left(e_{1}^{\frac{1}{(\beta+1)}} p_{1}^{\frac{\beta}{(\beta+1)}}+\ldots+e_{n}^{\frac{1}{(\beta+1)}} p_{n}^{\frac{\beta}{(\beta+1)}}\right)^{\frac{1}{\beta}}, \quad j=1, \ldots, n .
\end{aligned}
$$

\section{PROPORTIONALITY FOR THE INPUT-OUTPUT PRODUCTION FUNCTION}

In this section, we consider the case when an economy is described by an input-output production function:

$$
y(t)=\min \left\{\frac{x_{1}(t)}{c_{1}}, \ldots, \frac{x_{n}(t)}{c_{n}}\right\} .
$$

Here, the parameters $c_{j}, c_{j}>0$, specify productivity coefficients of the production factors $x_{j}$, $j=1, \ldots, n$.

For the balanced state of the economy, the proportionality conditions realizing the minimum in the input-output production function

$$
y=\frac{x_{1}}{c_{1}}, \ldots, \frac{x_{n}}{c_{n}}
$$

should be fulfilled.

The latter condition implies the proportionality of the production factors:

$$
c_{i k}=\frac{x_{i}}{x_{k}}=\frac{c_{i}}{c_{k}}, \quad i=1, \ldots, n, \quad k=1, \ldots, n,
$$

and specifies the balanced levels of the production factors $x_{j}=y c_{j}, j=1, \ldots, n$.

PROCEEDINGS OF THE STEKLOV INSTITUTE OF MATHEMATICS ～Vol. $293 \quad$ Suppl. 12016 
Taking into account cost constraints (2.1), one can derive the following condition for the optimal production:

$$
y=C \frac{1}{\left(p_{1} c_{1}+\ldots+p_{n} c_{n}\right)} .
$$

Thus, the proportionality conditions are an inherent property of the input-output production function.

\section{UNIVERSAL PRODUCTION FUNCTION}

Starting from this section, we pass to the second optimization level and consider a dynamic process; i.e., we assume that all the parameters of production functions, prices of production factors, and main variables (namely, production levels, costs of production factors, and consumption and investment levels) may depend on the time period $t, t \in\left[t_{0}, T\right], t_{0} \leq T \leq+\infty$.

Summarizing the conclusions from the previous sections on the proportionality conditions in the cost minimization problem and in the production maximization problem, we can establish a linear dependence of the optimal production $y(t)$ on the costs $C(t)$ and can call this dependence a universal production function of the model:

$$
y(t)=A(t) C(t)
$$

In addition, we can derive a linear dependence of the costs of the production factors $p_{j}(t) x_{j}(t)$, $j=1, \ldots, n$, on the costs $C(t)$ :

$$
p_{j}(t) x_{j}(t)=\gamma_{j}(t) C(t),
$$

with weight coefficients $\gamma_{j}=\gamma_{j}(t)$ satisfying the simplex relations

$$
\sum_{j=1}^{n} \gamma_{j}(t)=1, \quad \gamma_{j}(t) \geq 0, \quad j=1, \ldots, n .
$$

Here, the new scale coefficient $A=A(t)$ and the weight coefficients $\gamma_{j}=\gamma_{j}(t), j=1, \ldots, n$, are defined by the type of the production function.

Namely, for Cobb-Douglas production function (1.1), the scale coefficient $A$ is defined by the relation for the total factor productivity $a=a(t)$, by the prices $p_{j}=p_{j}(t)$, and by the elasticities $\alpha_{j}=\alpha_{j}(t), j=1, \ldots, n$ :

$$
A=A(t)=a(t)\left(\frac{\alpha_{1}(t)}{p_{1}(t)}\right)^{\alpha_{1}(t)} \ldots\left(\frac{\alpha_{n}(t)}{p_{n}(t)}\right)^{\alpha_{n}(t)}
$$

The weight coefficients $\gamma_{j}, j=1, \ldots, n$, for the Cobb-Douglas production function are given by the formulas

$$
\gamma_{j}(t)=\alpha_{j}(t), \quad j=1, \ldots, n .
$$

For CES production function (1.3), the scale coefficient $A=A(t)$ is determined by the following relation including the total factor productivity $e_{0}=e_{0}(t)$, the prices $p_{j}=p_{j}(t)$, the coefficient of substitution $\beta=\beta(t)$, and the share coefficients $e_{j}=e_{j}(t), j=1, \ldots, n$ :

$$
A=A(t)=e_{0}(t)\left(e_{1}(t)^{\frac{1}{(\beta(t)+1)}} p_{1}(t)^{\frac{\beta(t)}{(\beta(t)+1)}}+\ldots+e_{n}(t)^{\frac{1}{(\beta(t)+1)}} p_{n}(t)^{\frac{\beta(t)}{(\beta(t)+1)}}\right)^{-\frac{(\beta(t)+1)}{\beta(t)}} .
$$


The weight coefficients $\gamma_{j}, j=1, \ldots, n$, for the CES production function are given by the relations

$$
\gamma_{j}(t)=e_{j}(t)^{\frac{1}{(\beta(t)+1)}} p_{j}(t)^{\frac{\beta(t)}{(\beta(t)+1)}}\left(e_{1}(t)^{\frac{1}{(\beta(t)+1)}} p_{1}(t)^{\frac{\beta(t)}{(\beta(t)+1)}}+\ldots+e_{n}(t)^{\frac{1}{(\beta(t)+1)}} p_{n}(t)^{\frac{\beta(t)}{(\beta(t)+1)}}\right)^{-1} .
$$

In the case of input-output production function (3.1), the scale coefficient $A=A(t)$ is expressed in terms of the productivity coefficients $c_{j}=c_{j}(t)$ and the prices $p_{j}=p_{j}(t)$ according to the formulas

$$
A=A(t)=\frac{1}{\left(p_{1}(t) c_{1}(t)+\ldots+p_{n}(t) c_{n}(t)\right)} .
$$

The weight coefficients $\gamma_{j}, j=1, \ldots, n$, for the input-output production function are specified by the relations

$$
\gamma_{j}(t)=\frac{p_{j}(t) c_{j}(t)}{\left(p_{1}(t) c_{1}(t)+\ldots+p_{n}(t) c_{n}(t)\right)}, \quad j=1, \ldots, n
$$

\section{BALANCE EQUATIONS}

Let us introduce the following notation for the investment levels. The symbol $I=I(t)$ denotes the total investment, and the symbols $I_{j}=I_{j}(t)$ mean the levels of investment in the production factors $x_{j}=x_{j}(t), j=1, \ldots, n$. Thus, we have the balance relation for the investments:

$$
I(t)=I_{1}(t)+\ldots+I_{n}(t) .
$$

Introducing the relative investment levels with respect to the total production $y(t)$, we obtain for them the following connection:

$$
\begin{gathered}
s(t)=\frac{I(t)}{y(t)}, \quad s_{j}(t)=\frac{I_{j}(t)}{y(t)}, \quad s(t)=s_{1}(t)+\ldots+s_{n}(t), \\
0 \leq s(t)<1, \quad 0 \leq s_{j}(t) \leq s(t), \quad j=1, \ldots, n .
\end{gathered}
$$

The symbol $\mathrm{C}=\mathrm{C}(t)$ stands for the absolute consumption level.

Taking into account the balance equation for the investment and consumption, we get the relation $y(t)=\mathrm{C}(t)+I(t)=\mathrm{C}(t)+I_{1}(t)+\ldots+I_{n}(t)$. Similarly, passing to the relative variable for the consumption level $c(t)=\mathrm{C}(t) / y(t)$, we derive basic balance equations in the form

$$
1=c(t)+s(t)=c(t)+s_{1}(t)+\ldots+s_{n}(t) .
$$

\section{DYNAMICS OF THE MODEL AND THE UTILITY FUNCTION}

In this section, we describe the dynamics of the main variables of the model.

6.1. Dynamics of the production factors. Having the information on the prices $p_{j}=$ $p_{j}(t)$ and investment levels $I_{j}=I_{j}(t), j=1, \ldots, n$, one can recalculate the physical costs of the production factors:

$$
\Delta x_{j}(t)=\frac{I_{j}(t)}{p_{j}(t)}=\frac{s_{j}(t) y(t)}{p_{j}(t)} .
$$

We assume that the production factors $x_{j}=x_{j}(t)$ are subject to depreciation with specified coefficients $\delta_{j}=\delta_{j}(t)$. 
Further, treating the investments $\Delta x_{j}(t)$ in the production factor $j$ during the current period $t$ as control parameters, we can write differential equations for the dynamics of the production factors:

$$
\dot{x}_{j}(t)=\Delta x_{j}(t)-\delta_{j}(t) x_{j}(t), \quad j=1, \ldots, n .
$$

Using relation (6.1), which connects the costs of production factors and the production $y(t)$, we represent dynamics (6.2) in the following form:

$$
\dot{x}_{j}(t)=\frac{s_{j}(t) y(t)}{p_{j}(t)}-\delta_{j}(t) x_{j}(t), \quad j=1, \ldots, n .
$$

Based on relations (4.1) for the optimal value of production, we can derive equations for the dynamics of the production factors written in terms of the costs $C(t)$ :

$$
\dot{x}_{j}(t)=\frac{s_{j}(t) A(t) C(t)}{p_{j}(t)}-\delta_{j}(t) x_{j}(t), \quad j=1, \ldots, n .
$$

We can also obtain the dynamics of the production factors in the form standard for optimal control problems, using relation (4.2):

$$
\dot{x}_{j}(t)=x_{j}(t)\left(\frac{s_{j}(t)}{\gamma_{j}(t)} A(t)-\delta_{j}(t)\right), \quad j=1, \ldots, n .
$$

6.2. Dynamics of the costs. The equations describing the dynamics of the costs $C(t)$ are derived from the dynamics of production factors (6.3):

$$
\begin{gathered}
\dot{C}(t)=\dot{p}_{1}(t) x_{1}(t)+\ldots+\dot{p}_{n}(t) x_{n}(t)+p_{1}(t) \dot{x}_{1}(t)+\ldots+p_{n}(t) \dot{x}_{n}(t) \\
=\left(\frac{1}{p_{1}(t)} \dot{p}_{1}(t)-\delta_{1}(t)\right) p_{1}(t) x_{1}(t)+\ldots+\left(\frac{1}{p_{n}(t)} \dot{p}_{n}(t)-\delta_{n}(t)\right) p_{n}(t) x_{n}(t)+s(t) y(t) \\
=\left(\frac{1}{p_{1}(t)} \dot{p}_{1}(t)-\delta_{1}(t)\right) p_{1}(t) x_{1}(t)+\ldots+\left(\frac{1}{p_{n}(t)} \dot{p}_{n}(t)-\delta_{n}(t)\right) p_{n}(t) x_{n}(t)+s(t) A(t) C(t) .
\end{gathered}
$$

In view of the linear dependence of the costs of the production factors $p_{j}(t) x_{j}(t), j=1, \ldots, n$, on the total costs $C(t)$ (see (4.2)), we obtain the basic differential equation for the dynamics of the costs:

$$
\dot{C}(t)=C(t)\left(s(t) A(t)+\sum_{j=1}^{n} \gamma_{j}(t)\left(\frac{1}{p_{j}(t)} \dot{p}_{j}(t)-\delta_{j}(t)\right)\right) .
$$

Let us introduce the notation for the change rate (rise or fall) of the prices:

$$
r_{j}(t)=\frac{1}{p_{j}(t)} \dot{p}_{j}(t), \quad j=1, \ldots, n
$$

Based on the weights $\gamma_{j}(t), j=1, \ldots, n$, given by relations (4.3), we introduce the average price rate

$$
r(t)=\sum_{j=1}^{n} \gamma_{j}(t) r_{j}(t)
$$

and define the average depreciation coefficient

$$
\delta(t)=\sum_{j=1}^{n} \gamma_{j}(t) \delta_{j}(t)
$$


Using this notation, we obtain the final relation for the dynamics of the costs:

$$
\dot{C}(t)=C(t)(s(t) A(t)+(r(t)-\delta(t))) .
$$

Note that the only condition for the existence of solutions of the dynamics (6.6) of the costs that should be imposed on the dynamics of the prices $p_{j}(t)$, depreciation coefficients $\delta_{j}(t)$, and weight coefficients $\gamma_{j}(t), j=1, \ldots, n$, is the requirement of measurable dependence on time $t$.

Let us show that, for specified levels of the total investment $s(t)$, the dynamics of production factors (6.5) and the dynamics of total costs (6.6) determine uniquely the structure of investments in the production factors $s_{j}(t), j=1, \ldots, n$. More exactly, the following statement is valid.

Proposition 1. If the level of the total investment $s(t)$ is given, then the structure of investments in the production factors $s_{j}(t), j=1, \ldots, n$, which is necessary to provide the proportionality in the dynamics of the model, is recalculated by the relations

$$
s_{j}(t)=\gamma_{j}(t) s(t)+\frac{\gamma_{j}(t)}{A(t)}\left(\left(r(t)-r_{j}(t)\right)-\left(\delta(t)-\delta_{j}(t)\right)\right)+\frac{1}{A(t)} \dot{\gamma}_{j}(t), \quad j=1, \ldots, n .
$$

Proof. Consider the dynamics of the factors (6.3), which implies that

$$
p_{j}(t) \dot{x}_{j}(t)=s_{j}(t) A(t) C(t)-\delta_{j}(t) \gamma_{j}(t) C(t), \quad j=1, \ldots, n .
$$

Using formula (4.2) for the costs of production factors, we can derive relations for the growth rates of the factors:

$$
p_{j}(t) \dot{x}_{j}(t)=\gamma_{j}(t) \dot{C}(t)+C(t) \dot{\gamma}_{j}(t)-C(t) \gamma_{j}(t) r_{j}(t), \quad j=1, \ldots, n .
$$

Taking into account the dynamics of the costs (6.6), we obtain from formula (6.9) the following expression:

$$
p_{j}(t) \dot{x}_{j}(t)=\gamma_{j}(t) C(t)((r(t)-\delta(t))+s(t) A(t))-C(t) \gamma_{j}(t) r_{j}(t)+C(t) \dot{\gamma}_{j}(t), \quad j=1, \ldots, n .
$$

Excluding similar terms in formulas (6.8) and (6.10), we obtain required relations (6.7) for the structure of investments in production factors.

Remark 1. Note that, if obtained investment levels (6.7) are nonnegative $\left(s_{j}(t) \geq 0, j=\right.$ $1, \ldots, n)$, then these investments can be implemented based on the current production $y(t)$; in this sense, they should be called feasible investments.

However, the negative signs of the investment levels $\left(s_{j}(t)<0, j=1, \ldots, n\right)$ are also possible. Here, we should say that, in principle, negative levels can be allowed in the model. We mean that it is possible to invest in a production factor $x_{j}$ not only due to the production $y(t)$ obtained in the current period but also using the stocks of other production factors $x_{k}, k=1, \ldots, n, k \neq j$.

Remark 2. It is important that, according to dynamics (6.4), the trajectories of the production factors are nonnegative, $x_{j}(t) \geq 0, j=1, \ldots, n$, on the whole time interval $t \in\left[t_{0}, T\right]$, irrespective of the signs of investments $s_{j}(t), j=1, \ldots, n$.

6.3. Constraints on the investment and consumption. We assume that there exists a constraint on the lower consumption level $c^{0}$ (the living wage) measured as a percentage of the production:

$$
0<c_{0} \leq c(t) \leq 1
$$

From balance equation (5.1), it follows that there is an upper possible investment level $s^{0}$ :

$$
0 \leq s(t) \leq s^{0}<1, \quad s^{0}=1-c^{0} .
$$


6.4. Utility function. Let us introduce a utility function to examine the quality of model trajectories at the second optimization level. Toward this aim, we use the integral logarithmic index of discounted consumption, which, according to balance equation (5.1) and the structure of universal production function (4.1), can be presented by the relations

$J=\int_{t_{0}}^{T} e^{-\rho t} \ln c(t) d t=\int_{t_{0}}^{T} e^{-\rho t}(\ln y(t)+\ln (1-s(t))) d t=\int_{t_{0}}^{T} e^{-\rho t}(\ln A(t)+\ln C(t)+\ln (1-s(t))) d t$.

Here, the parameter $\rho, \rho>0$, specifies the discount rate.

\section{OPTIMAL CONTROL PROBLEM}

Consider the optimal control problem for the investment process at the second optimization level. The statement of this problem implies the maximization in the controlled system of the utility function

$$
J(C(\cdot), s(\cdot), T)=\int_{t_{0}}^{T} e^{-\rho t}(\ln A(t)+\ln C(t)+\ln (1-s(t))) d t
$$

on trajectories generated by the dynamics

$$
\dot{C}(t)=C(t)(A(t) s(t)-\sigma(t)) .
$$

Here, the parameter of "generalized" cost depreciation $\sigma=\sigma(t)$ is defined by the formula

$$
\sigma(t)=\delta(t)-r(t)
$$

The control investment parameter is subject to the constraints

$$
0 \leq s(t) \leq s^{0}<1
$$

The phase variable $C(t)$ of system (7.2) satisfies the initial conditions

$$
C\left(t_{0}\right)=C_{0}
$$

Note that problem (7.1)-(7.4) is a classical optimal control problem both with finite horizon $T<+\infty$ and with infinite horizon $T=+\infty$ (see $[7,8])$.

\section{OPTIMAL SOLUTIONS}

In this section, we consider the application of the Pontryagin maximum principle equipped with transversality conditions to solving the formulated optimal control problem.

PROCEEDINGS OF THE STEKLOV INSTITUTE OF MATHEMATICS $\quad$ Vol. $293 \quad$ Suppl. $1 \quad 2016$ 
8.1. Hamiltonian functions of optimal control problems. Let us start to solve the optimal control problem with compiling the Hamiltonian function of controlled system (7.1)-(7.4):

$$
\tilde{H}(t, C(t), s(t), \tilde{\psi}(t))=e^{-\rho t}(\ln A(t)+\ln C(t)+\ln (1-s(t)))+\tilde{\psi}(t) C(t)(s(t) A(t)-\sigma(t)) .
$$

Here, the parameter $\tilde{\psi}=\tilde{\psi}(t)$ stands for the adjoint variable.

Using the substitution for the adjoint variable

$$
\psi(t)=e^{\rho t} \tilde{\psi}(t)
$$

we obtain the stationary (not discounted) Hamiltonian

$$
H(t, C(t), s(t), \psi(t))=\ln A(t)+\ln C(t)+\ln (1-s(t))+\psi(t) C(t)(s(t) A(t)-\sigma(t)),
$$

which is connected with the original Hamiltonian by the relation

$$
\tilde{H}(t, C(t), s(t), \tilde{\psi}(t))=e^{-\rho t} H(t, C(t), s(t), \psi(t)) .
$$

Lemma 1. The Hamiltonian $H(t, C, s, \psi)(8.2)$ is a strictly concave function in the variables $C$ and $s$ for all values of the variables $t$ and $\psi$.

Proof. The proof of the statement follows immediately from the fact that the matrix of second derivatives of Hamiltonian (8.2) is strictly negative definite. This property is verified by means of Sylvester's criterion in the variables $C$ and $s$ for all values of the variables $t$ and $\psi$.

8.2. The Pontryagin maximum principle. Note that the conditions of the existence theorem (see $[8,11]$ ) are fulfilled for control problem $(7.1)-(7.4)$. Moreover, we can formulate the necessary optimality conditions for the control problems with finite horizon [7] and with infinite horizon $[8,14]$ in the form of the Pontryagin maximum principle.

Theorem 1. Let $\left(s^{*}, C^{*}\right)$ be an optimal process in the control problem. Then, there exists an adjoint variable $\tilde{\psi}$ corresponding to the process $\left(s^{*}, C^{*}\right)$ and satisfying the adjoint equation

$$
\dot{\tilde{\psi}}(t)=-\frac{\partial \tilde{H}}{\partial C}\left(t, C^{*}(t), s^{*}(t), \tilde{\psi}(t)\right)
$$

such that

(1) the process $\left(s^{*}, C^{*}\right)$ and the adjoint variable $\tilde{\psi}$ satisfy the condition of the Pontryagin maximum principle:

$$
\tilde{H}\left(t, C^{*}, s^{*}, \tilde{\psi}\right)=\max \left\{\tilde{H}\left(t, C^{*}, s, \tilde{\psi}\right), s \in\left[0, s^{0}\right]\right\}
$$

(2) the adjoint variable $\tilde{\psi}$ takes strictly positive values:

$$
\tilde{\psi}(t)>0 \quad \forall t, \quad t_{0} \leq t<T, \quad T \leq+\infty
$$

(3) the adjoint variable $\tilde{\psi}$ satisfies the transversality condition for the problem with finite horizon:

$$
\tilde{\psi}(T)=0
$$

and for the problem with infinite horizon:

$$
\lim _{t \rightarrow \infty} \tilde{\psi}(t) C^{*}(t)=0
$$


Remark 3. Note that, for the Hamiltonian $H(t, C, s, \psi)(8.2)$, the dynamics of adjoint variable $\psi(t)(8.1)$ is described by the differential equation

$$
\dot{\psi}(t)=\rho \psi(t)-\frac{\partial H}{\partial C}\left(t, C^{*}(t), s^{*}(t), \psi(t)\right) .
$$

In this case, the maximum condition has the form

$$
H\left(t, C^{*}, s^{*}, \tilde{\psi}\right)=\max \left\{H\left(t, C^{*}, s, \tilde{\psi}\right), s \in\left[0, s^{0}\right]\right\} .
$$

The adjoint variable $\psi$ is strictly positive:

$$
\psi(t)>0 \quad \forall t, \quad t_{0} \leq t<T, \quad T \leq+\infty .
$$

The transversality condition is given by the relation

$$
\psi(T)=0
$$

for the problem with finite horizon, and it is represented in the form

$$
\lim _{t \rightarrow \infty} e^{-\rho t} \psi(t) C^{*}(t)=0
$$

for the problem with infinite horizon.

8.3. Maximized Hamiltonian. Let us calculate the values of the maximized Hamiltonian in optimal control problem (7.1)-(7.4):

$$
\begin{gathered}
\bar{H}(t, C(t), \psi(t))=\max _{0 \leq s \leq s^{0}} \hat{H}(t, C(t), s, \psi(t)) \\
=\ln A(t)+\ln C(t)-\psi(t) C(t) \sigma(t)+\max _{0 \leq s \leq s^{0}}\{\ln (1-s)+\psi(t) C(t) A(t) s\} .
\end{gathered}
$$

8.4. Structure of optimal control. Using the necessary optimality conditions, we can obtain the structure of the optimal control, which provides the maximum value of Hamiltonian (8.7):

$$
s= \begin{cases}0, & 1-\frac{1}{\psi(t) A(t) C(t)}<0 \\ 1-\frac{1}{\psi(t) A(t) C(t)}, & 0 \leq 1-\frac{1}{\psi(t) A(t) C(t)} \leq s^{0} \\ s^{0}, & 1-\frac{1}{\psi(t) A(t) C(t)}>s^{0} .\end{cases}
$$

Remark 4. Note that solution (8.8) of the necessary optimality conditions is indeed the unique maximum point due to the fact (established in Lemma 1) that the Hamiltonian $\hat{H}(t, C, s, \psi)(8.2)$ is strictly concave in the variable $s$.

8.5. Structure of the maximized Hamiltonian. The relations for the optimal control given by (8.8) define the structure of the maximized Hamiltonian; the latter is specified by three branches. The first branch corresponds to the zero extremal control regime, $s_{1}=0$ :

$$
\begin{aligned}
& \bar{H}_{1}(t, C(t), \psi(t))=\ln A(t)+\ln C(t)-\psi(t) C(t) \sigma(t), \\
& 1-\frac{1}{\psi(t) A(t) C(t)}<0 .
\end{aligned}
$$

PROCEEDINGS OF THE STEKLOV INSTITUTE OF MATHEMATICS ～Vol. $293 \quad$ Suppl. 122016 
The second branch corresponds to the regular control regime, $s_{2}=1-1 /(\psi(t) A(t) C(t))$ :

$$
\begin{aligned}
& \bar{H}_{2}(t, C(t), \psi(t))=-\ln \psi(t)+\psi(t) C(t)(A(t)-\sigma(t))-1, \\
& 0 \leq 1-\frac{1}{\psi(t) A(t) C(t)} \leq s^{0} .
\end{aligned}
$$

The third branch is determined by the extremal regime of the highest control level, $s_{3}=s^{0}$ :

$$
\begin{aligned}
& \bar{H}_{3}(t, C(t), \psi(t))=\ln A(t)+\ln C(t)+\ln \left(1-s^{0}\right)+\psi(t) C(t)\left(A(t) s^{0}-\sigma(t)\right), \\
& 1-\frac{1}{\psi(t) A(t) C(t)}>s^{0} .
\end{aligned}
$$

It turns out that the maximized Hamiltonian $\bar{H}(t, C, \psi)(8.7)$ is concave in the variable $C$; this is established below.

Lemma 2. The maximized Hamiltonian $\bar{H}(t, C, \psi)(8.7)$ is concave in the variable $C$.

Proof. One can obtain the result of Lemma 2 from the fact that all three branches $\bar{H}_{i}(t, C, \psi)$, $i=1,2,3$, are concave functions in the variable $C$, which are smoothly (with continuous derivatives) joined together generating the maximized Hamiltonian $\bar{H}(t, C, \psi)$ for all fixed values of the variables $t$ and $\psi$.

8.6. Hamiltonian systems. According to the structure of the maximized Hamiltonian $\bar{H}(t, C, \psi)(8.7)$, we can construct a family of three Hamiltonian systems, $i=1,2,3$, defined by conditions (8.3), (8.4) of the Pontryagin maximum principle:

$$
\left\{\begin{array}{l}
\dot{C}(t)=C(t)\left(A(t) s_{i}(t)-\sigma(t)\right), \\
\dot{\psi}(t)=\rho \psi(t)-\frac{\partial H_{i}}{\partial C}(t, C(t), \psi(t)) .
\end{array}\right.
$$

Here, the derivatives of the maximized Hamiltonian $\bar{H}(t, C, \psi)(8.7)$ are calculated in accordance with its branches, $i=1,2,3$ :

$$
\frac{\partial \bar{H}_{i}}{\partial C}(t, C(t), \psi(t))= \begin{cases}\frac{1}{C(t)}-\sigma(t) \psi(t), & i=1, \\ (A(t)-\sigma(t)) \psi(t), & i=2, \\ \frac{1}{C(t)}+\left(A(t) s^{0}-\sigma(t)\right) \psi(t), & i=3 .\end{cases}
$$

8.7. Analysis of Hamiltonian systems. Let us introduce a new variable $x=x(t)$, which means generalized costs of the production factors in the Pontryagin maximum principle:

$$
x=x(t)=C(t) \psi(t) .
$$

One can derive the equation of Hamiltonian dynamics for the generalized costs $x(t)$.

Proposition 2. According to Hamiltonian dynamics (8.9), the generalized costs $x(t)$ satisfy the differential equation

$$
\dot{x}(t)=\rho x(t)-1 \text {. }
$$

Proof. The proof of this statement immediately follows from the fact that, according to relations (8.10), which define all branches of the Hamiltonian dynamics, $i=1,2,3$, there is the following chain of equalities:

$\dot{x}(t)=\dot{C}(t) \psi(t)+C(t) \dot{\psi}(t)=\left(\rho-\sigma(t)+A(t) s_{i}(t)\right) C(t) \psi(t)-C(t) \frac{\partial H_{i}}{\partial C}(t, C(t), \psi(t))=\rho x(t)-1$. 
One can also obtain the general solution of equation (8.11) describing the dynamics of generalized costs.

Proposition 3. The general solution for the generalized costs subject to Hamiltonian dynamics (8.9) is represented by the relation

$$
x(t)=\frac{1}{\rho}+B e^{\rho t} .
$$

Here, the symbol $B$ denotes a constant that can be found either from transversality conditions (8.5) for the problem with finite horizon $B=-\frac{e^{-\rho T}}{\rho}$ or from transversality conditions (8.6) for the problem with infinite horizon $B=0$.

Proof. The proof of this statement follows from the Cauchy formula for differential equation (8.11).

Remark 5. Note that the solution of Hamiltonian dynamics equation (8.9) for the generalized costs is described by the relation

$$
x(t)=\frac{1}{\rho}\left(1-e^{\rho(t-T)}\right)
$$

for the problem with finite horizon and by the formula

$$
x(t)=\frac{1}{\rho}
$$

for the problem with infinite horizon.

8.8. Optimal control. Now, we obtain analytic relations for the optimal open-loop control. In order to do this, it is necessary to substitute solutions (8.12) and (8.13) of Hamiltonian dynamics equation (8.9) into the structure of optimal control (8.8).

In the case of the problem with finite horizon, the optimal open-loop control is expressed in the form

$$
s^{*}(t)= \begin{cases}0, & 1-\frac{\rho}{A(t)\left(1-e^{\rho(t-T)}\right)}<0 ; \\ 1-\frac{\rho}{A(t)\left(1-e^{\rho(t-T)}\right)}, & 0 \leq 1-\frac{\rho}{A(t)\left(1-e^{\rho(t-T)}\right)} \leq s^{0} \\ s^{0}, & 1-\frac{\rho}{A(t)\left(1-e^{\rho(t-T)}\right)}>s^{0} .\end{cases}
$$

In the case of the problem with infinite horizon, the optimal open-loop control is described by the relations

$$
s^{*}(t)= \begin{cases}0, & 1-\frac{\rho}{A(t)}<0 \\ 1-\frac{\rho}{A(t)}, & 0 \leq 1-\frac{\rho}{A(t)} \leq s^{0} \\ s^{0}, & 1-\frac{\rho}{A(t)}>s^{0} .\end{cases}
$$

Note that, given the optimal investments $s^{*}(t)$, we can make a reverse transfer from the second optimization level to the first one and can determine the structure of optimal investments $s_{j}^{*}(t)$ in production factors according to the proportionality conditions. We can formulate the following result more exactly. 
Proposition 4. If the optimal investments $s^{*}=s^{*}(t)$ are determined either by relation (8.14) for the problem with finite horizon or by relation (8.15) for the problem with infinite horizon, then the optimal investments $s_{j}^{*}(t)$ in production factors, which sustain the proportional development of the system, are recalculated by formulas (6.7) in the following form:

$$
s_{j}^{*}(t)=\gamma_{j}(t) s^{*}(t)+\frac{\gamma_{j}(t)}{A(t)}\left(\left(r(t)-r_{j}(t)\right)-\left(\delta(t)-\delta_{j}(t)\right)\right)+\frac{1}{A(t)} \dot{\gamma}_{j}(t), \quad j=1, \ldots, n .
$$

Let us make an important remark on the structure of optimal control (8.14) for the problem with finite horizon.

Remark 6. If the scale coefficient $A=A(t)$ (4.1) in the universal production function satisfies the boundedness property in the problem with finite horizon $T$, i.e.,

$$
0<A(t) \leq A^{0}, \quad t_{0} \leq t \leq T,
$$

then there exists a nonempty time interval

$$
T-\frac{1}{\rho} \ln \left(\frac{A^{0}}{A^{0}-\rho}\right) \leq t \leq T,
$$

where the optimal investments $s^{*}(t)$ take zero values:

$$
s^{*}(t) \equiv 0
$$

Therefore, in problems with finite horizon, the optimal investments inevitably degenerate to zero levels, whereas the product should be completely consumed, $y(t)=\mathrm{C}(t)$, on some time interval close to the termination time $T$ of the control process.

8.9. Sufficient optimality conditions in the maximum principle. Let us formulate a result that provides sufficient optimality conditions for trajectories satisfying the Pontryagin maximum principle.

Proposition 5. In virtue of the concavity of maximized Hamiltonian $\bar{H}(t, C, \psi)(8.7)$ in the variable $C$ obtained in Lemma 2, the Pontryagin maximum principle sorts out trajectories that are optimal for control problem (7.1)-(7.4).

Proof. This statement is proved by analogy with the argument proposed in [14].

\section{ACKNOWLEDGMENTS}

This work was supported by the Russian Science Foundation (project no. 15-11-10018).

\section{REFERENCES}

1. K. J. Arrow, Production and Capital: Collected Papers (Belknap Harvard, 1985), Vol. 5.

2. R. J. Barro and X. Sala-i-Martin, Economic Growth (McGraw-Hill, New York, 1995).

3. G. M. Grossman and E. Helpman, Innovation and Growth in the Global Economy (MIT, Cambridge, MA, 1991).

4. K. Shell, "Applications of Pontryagin's maximum principle to economics," in Mathematical Systems Theory and Economics (Springer, Berlin, 1969), Vol. 1, pp. 241-292.

5. R. M. Solow, Growth Theory: An Exposition (Oxford Univ. Press, New York, 1970).

PROCEEDINGS OF THE STEKLOV INSTITUTE OF MATHEMATICS ～Vol. $293 \quad$ Suppl. 122016 
6. M. Intriligator, Mathematical Optimization and Economic Theory (Prentice-Hall, Englewood Cliffs, NJ, 1971; Airis, Moscow, 2002).

7. L. S. Pontryagin, V. G. Boltyanskii, R. V. Gamkrelidze, and E. F. Mishchenko, The Mathematical Theory of Optimal Processes (Fizmatgiz, Moscow, 1961; Wiley, New York, 1962).

8. S. M. Aseev and A. V. Kryazhimskii, "The Pontryagin maximum principle and optimal economic growth problems," Proc. Steklov Inst. Math. 257, 1-255 (2007).

9. S. M. Aseev and V. M. Veliov, "Maximum principle for infinite-horizon optimal control problems with dominating discount," Dyn. Contin. Discrete Impuls. Syst., Ser. B: Appl. Algorithms 19 (1-2), 43-63 (2012).

10. M. S. Nikol'skii, "Investigation of the continuity and Lipschitz properties for the Bellman function in some

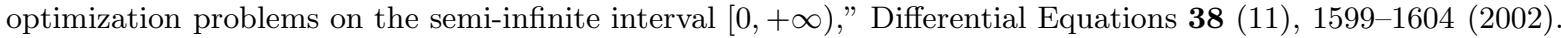

11. E. J. Balder, "An existence result for optimal economic growth problems," J. Math. Anal. Appl. 95, 195-213 (1983).

12. A. M. Tarasyev and C. Watanabe, "Optimal dynamics of innovation in models of economic growth," J. Math. Anal. Appl. 108, 175-203 (2001).

13. A. V. Kryazhimskiy and C. Watanabe, Optimization of Technological Growth (Gendaitosho, Kanagawa, 2004).

14. A. A. Krasovskii and A. M. Tarasyev, "Properties of Hamiltonian systems in the Pontryagin maximum principle for economic growth problems," Proc. Steklov Inst. Math. 262, 121-138 (2008).

15. A. A. Krasovskii and A. M. Tarasyev, "An algorithm for construction of optimal timing solutions in problems with a stochastic payoff function," Appl. Math. Comput. 204 (2), 632-643 (2008).

16. A. A. Krasovskii, A. V. Kryazhimskiy, and A. M. Tarasyev, "Optimal control design in models of economic growth," in Evolutionary Methods for Design, Optimization and Control, Ed. by P. Neittaanmaki, J. Periaux, and T. Tuovinen (CIMNE, Barcelona, 2008), pp. 70-75.

17. Dynamic Systems, Economic Growth, and the Environment, Ed. by J. Crespo Cuaresma, T. Palokangas, and A. Tarasyev, (Springer, New York, 2010), Ser. Dynamic Modeling and Econometrics in Economics and Finance, Vol. 12.

18. Green Growth and Sustainable Development, Ed. by J. Crespo Cuaresma, T. Palokangas, and A. Tarasyev, (Springer, New York, 2013), Ser. Dynamic Modeling and Econometrics in Economics and Finance, Vol. 14.

19. A. M. Tarasyev and A. A. Usova, "Stabilizing the Hamiltonian system for constructing optimal trajectories," Proc. Steklov Inst. Math. 277, 248-265 (2012).

20. A. M. Taras'ev, A. A. Usova, O. V. Russkikh, and W. Wang, "Optimal trajectory construction by integration of Hamiltonian dynamics in models of economic growth under resource constraints," Trudy Inst. Mat. Mekh. UrO RAN 20 (4), 258-276 (2014).

21. A. M. Tarasyev, A. A. Usova, and Yu. V. Shmotina, "Projection of the Russian economic development in the framework of the optimal control model by investments in fixed assets," Ekon. Regiona, No. 3, $265-273$ (2014).

22. B. S. Jensen, P. K. Alsholm, M. E. Larsen, and J. M. Jensen, "Dynamic structure, exogeneity, phase portraits, growth paths, and scale and substitution elasticities," Rev. Internat. Econ. 13 (1), 59-89 (2005).

23. J. Hofbauer and K. Sigmund, The Theory of Evolution and Dynamical Systems (Cambridge Univ. Press, Cambridge, 1988).

24. A. V. Kryazhimskii and Yu. S. Osipov, "On differential-evolutionary games," Proc. Steklov Inst. Math. 211, 234-261 (1995).

25. N. N. Krasovskii and A. I. Subbotin, Positional Differential Games (Nauka, Moscow, 1974) [in Russian].

Translated by V. Rozenberg 